\title{
Improving preparedness to respond to cross-border hepatitis A outbreaks in the European Union/European Economic Area: towards comparable sequencing of hepatitis A virus
}

Theresa Enkirch ${ }^{1,2}$, Ettore Severi3,4, Harry Vennema5, Lelia Thornton', Jonathan Dean ${ }^{7}$, Maria-Louise Borg ${ }^{8}$, Anna Rita

Ciccaglione9, Roberto Bruni9, Iva Christova ${ }^{10}$, Siew Lin Ngui ${ }^{11}$, Koye Balogun ${ }^{11}$, Vratislav Němeček ${ }^{12}$, Mia Kontio ${ }^{13}$, Mária Takács ${ }^{14}$, Andrea Hettmann ${ }^{14}$, Rita Korotinska ${ }^{15}$, Arthur Löve ${ }^{16}$, Ana Avellón ${ }^{17}$, Milagros Muñoz-Chimeno ${ }^{17}$, Rita de Sousa ${ }^{18}$, Denisa Janta ${ }^{19}$, Jevgenia Epštein ${ }^{20}$, Sofieke Klamer ${ }^{21}$, Vanessa Suin ${ }^{22}$, Stephan W Aberle ${ }^{23}$, Heidemarie Holzmann ${ }^{23}$, Kassiani Mellou ${ }^{24}$, Josefine Lundberg Ederth ${ }^{1}$, Lena Sundqvist ${ }^{1}$, Anne-Marie Roque-Afonso ${ }^{25}$, Sanja Kurečić Filipović ${ }^{26}$, Mario Poljak ${ }^{27}$, Line Vold ${ }^{28}$, Kathrine Stene-Johansen ${ }^{28}$, Sofie Midgley ${ }^{29}$, Thea Kølsen Fischer ${ }^{29,30}$, Mirko Faber ${ }^{31}$, Jürgen J Wenzel ${ }^{32}$, Johanna Takkinen ${ }^{3}$, Katrin Leitmeyer ${ }^{3}$

1. Public Health Agency of Sweden, Solna, Sweden

2. European Programme for Public Health Microbiology Training (EUPHEM), European Centre for Disease Prevention and Control (ECDC), Solna, Sweden

3. European Centre for Disease Prevention and Control (ECDC), Solna, Sweden

4. Karolinska Institutet, Stockholm, Sweden

5. National Institute for Public Health and the Environment (RIVM), Bilthoven, the Netherlands

6. HSE - Health Protection Surveillance Centre, Dublin, Ireland

7. National Virus Reference Laboratory, Dublin, Ireland

8. 8 Ministry of Health, Msida, Malta

9. National Institute of Health, Rome, Italy

10. National Center of Infectious and Parasitic Diseases, Sofia, Bulgaria

11. Public Health England (PHE), London, United Kingdom

12. National Institute of Public Health, Prague, Czech Republic

13. National Institute for Health and Welfare (THL), Helsinki, Finland

14. National Public Health Institute, Budapest, Hungary

15. Centre for Disease Prevention and Control, Riga, Latvia

16. Landspitali- National University Hospital, Reykjavik, Iceland

17. Carlos III Institute of Health, Madrid, Spain

18. National Institute of Health Dr. Ricardo Jorge (INSA), Lisbon, Portugal

19. National Institute of Public Health, Bucharest, Romania

20. Health Board, Tallinn, Estonia

21. Scientific Institute of Public Health, Brussels, Belgium

22. Sciensano, Directorate Infectious diseases in humans, Brussels, Belgium

23. Center for Virology, Medical University of Vienna, Vienna, Austria

24. Hellenic Centre for Disease Control and Prevention, Athens, Greece

25. National Reference Centre for Hepatitis A, Villejuif, France

26. Croatian Institute of Public Health, Zagreb, Croatia

27. Institute of Microbiology and Immunology, Ljubljana, Slovenia

28. Norwegian institute of Public Health, Oslo, Norway

29. Statens Serum Institut (SSI), Copenhagen, Denmark

30. Department of Infectious Diseases and Global Health, University of Southern Denmark, Odense, Denmark

31. Robert Koch Institute (RKI), Berlin, Germany

32. National Reference Laboratory for HAV, Regensburg University Medical Center, Regensburg, Germany

Correspondence: Theresa Enkirch(Theresa.Enkirch@folkhalsomyndigheten.se)

Citation style for this article:

Enkirch Theresa, Severi Ettore, Vennema Harry, Thornton Lelia, Dean Jonathan, Borg Maria-Louise, Ciccaglione Anna Rita, Bruni Roberto, Christova Iva, Ngui

Siew Lin, Balogun Koye, Němeček Vratislav, Kontio Mia, Takács Mária, Hettmann Andrea, Korotinska Rita, Löve Arthur, Avellón Ana, Muñoz-Chimeno Milagros, de

Sousa Rita, Janta Denisa, Epštein Jevgenia, Klamer Sofieke, Suin Vanessa, Aberle Stephan W., Holzmann Heidemarie, Mellou Kassiani, Ederth Josefine Lundberg,

Sundqvist Lena, Roque-Afonso Anne-Marie, Filipović Sanja Kurečić, Poljak Mario, Vold Line, Stene-Johansen Kathrine, Midgley Sofie, Fischer Thea Kølsen, Faber

Mirko, Wenzel Jürgen J., Takkinen Johanna, Leitmeyer Katrin. Improving preparedness to respond to cross-border hepatitis A outbreaks in the European Union/

European Economic Area: towards comparable sequencing of hepatitis A virus. Euro Surveill. 2019;24(28):pii=1800397. https://doi.org/10.2807/1560-7917.

ES.2019.24.28.1800397

Introduction: Sequence-based typing of hepatitis A virus (HAV) is important for outbreak detection, investigation and surveillance. In 2013, sequencing was central to resolving a large European Union (EU)wide outbreak related to frozen berries. However, as the sequenced HAV genome regions were only partly comparable between countries, results were not always conclusive. Aim: The objective was to gather information on HAV surveillance and sequencing in EU/European Economic Area (EEA) countries to find ways to harmonise their procedures, for improvement of cross-border outbreak responses. Methods: In 2014, the European Centre for Disease Prevention and Control (ECDC) conducted a survey on HAV surveillance practices in EU/EEA countries. The survey enquired whether a referral system for confirming primary diagnostics of hepatitis $A$ existed as well as a central collection/storage of hepatitis A cases' samples for typing. Questions on HAV sequencing procedures were also asked. Based on the results, an expert 
consultation proposed harmonised procedures for cross-border outbreak response, in particular regarding sequencing. In 2016, a follow-up survey assessed uptake of suggested methods. Results: Of 31 EU/ EEA countries, 23 (2014) and 27 (2016) participated. Numbers of countries with central collection and storage of HAV positive samples and of those performing sequencing increased from 12 to 15 and 12 to 14 respectively in 2016, with all countries typing an overlapping fragment of $218 \mathrm{nt}$. However, variation existed in the sequenced genomic regions and their lengths. Conclusions: While HAV sequences in EU/EEA countries are comparable for surveillance, collaboration in sharing and comparing these can be further strengthened.

\section{Introduction}

The hepatitis A virus (HAV) of the Picornaviridae family affects 114 million people annually and is a common cause of food-borne infections worldwide $[1,2]$. It is mainly transmitted through faecal-oral route via person-to-person contact or contaminated food and water [3]. Symptoms range from fever, nausea, diarrhoea, abdominal pain and jaundice to acute liver failure. In children however, the majority of HAV infections are subclinical and asymptomatic [4].

Hepatitis $A$ is a notifiable disease in all countries of the European Union/European Economic Area (EU/EEA) [5]. Most EU/EEA countries, with the exception of few eastern EU countries, have experienced hepatitis A notification rates below two cases per 100,000 population in the last two decades or more [6]. Consequently, and based on World Health Organization (WHO) classification, the HAV endemicity is currently low or very low with a resulting high population susceptibility $[1,7]$. In line with WHO guidance, in most EU/EEA countries with low or very low endemicity, hepatitis A vaccination is recommended only to individuals at increased risk of infection, including travellers to endemic countries, people who inject drugs (PWID) and men who have sex with men (MSM). However, due to suboptimal vaccination uptakes, travel to countries with high or intermediate HAV endemicity is a common risk factor for infection [8] and multi-country outbreaks have been reported since 2012 among MSM and PWID $[9,10]$. Starting 2016, a large outbreak disproportionally affecting MSM was identified in Europe [11-13]. Between June 2016 and May 2017, ca 1,400 hepatitis A cases in $16 \mathrm{EU} / \mathrm{EEA}$ countries were identified, with genome sequencing showing concomitant circulation of three genetically distinct HAV strains $[14,15]$. In the same period, several other HAV outbreaks affecting MSM were reported from the United States, Israel and Chile [16]. In recently published studies, sequencing demonstrated a link between the cases in Israel and Chile and the ongoing EU outbreak $[17,18]$. Also a large food-borne outbreak caused by berries was reported in the EU/EEA in 2013-14, with sequencing being key in identifying the vehicle of infection $[19,20]$.
Sequence-based typing together with conventional epidemiological methods play a crucial role in outbreak investigations by confirming or refuting transmission chains, monitoring circulating strains and documenting introduction of new strains [20]. The combined molecular and epidemiological methods provide important information for action, allowing rapid implementation of public health measures such as vaccination of risk groups or identification of contaminated vehicles of infection, which can be traced back and forward and, when these are commercialised, taken off the market to avoid new infections. To respond to multi-country hepatitis $A$ outbreaks, similar sequencing methods (same amplified target region, including length, for sequencing and phylogenetic analysis) and collaborations and exchange of HAV sequence information between EU/ EEA countries offer substantial benefits. This was demonstrated during the large 2013-14 EU-wide outbreak related to frozen berries, although sequencing results were not always comparable as different amplified regions of the HAV genome were used for typing in some of the countries $[19,20]$.

The HAV genome consists of linear positive singlestranded RNA and has a single open reading frame (ORF) divided into three functional regions, $P_{1}-P_{3} . P_{1}$ encodes the capsid polypeptides (viral protein VP1-4) and $\mathrm{P}_{2}$ and $\mathrm{P}_{3}$ encode the non-structural polyproteins $2 A-2 C$ and $3 A-3 D$ required for virus replication. Only one single serotype has been described but six HAV genotypes are defined. The genotypes differ by $15-25 \%$ in the $n t$ sequence of the $\mathrm{VP}_{1} / 2 \mathrm{~A}$ region and are further divided into subtypes (7.0-7.5\% difference). Subtypes A and B of genotype I, II and III are infectious for humans [21-24]. In Europe, subgenotype IA is the most predominant subgenotype [25]. The presence of only one known serotype can partially be explained by the low mutation rate of HAV and the low variability resulting in a well-conserved polyprotein [26].

After the large 2013-14 berry-related EU-wide outbreak, the recognition of the importance of sequencing in terms of outbreak investigations and preparedness led ECDC to convene in October 2014 a multidisciplinary expert meeting. The aim was to discuss the need for agreement on a common genomic region for sequencing and/or the possible use of a protocol that HAVNET, a global network of hepatitis A laboratories for sharing molecular and epidemiological data on hepatitis A cases [27], had developed. In preparation of this meeting, ECDC performed a survey among the EU/EEA countries in order to collect information on HAV genotyping practices, including whether primary diagnostic samples of patients with hepatitis $A$ could be referred for confirmation or genotyping and whether such samples were centrally collected. The survey also aimed to identify the most suitable common target region for sequencing, and the existence of collaborations between the public health sector with HAVNET and with the food sector. Based on the findings of this first survey, it was agreed to recommend 


\section{FIGURE 1}

Overview of responses to surveyed questions by country, 2014 and 2016

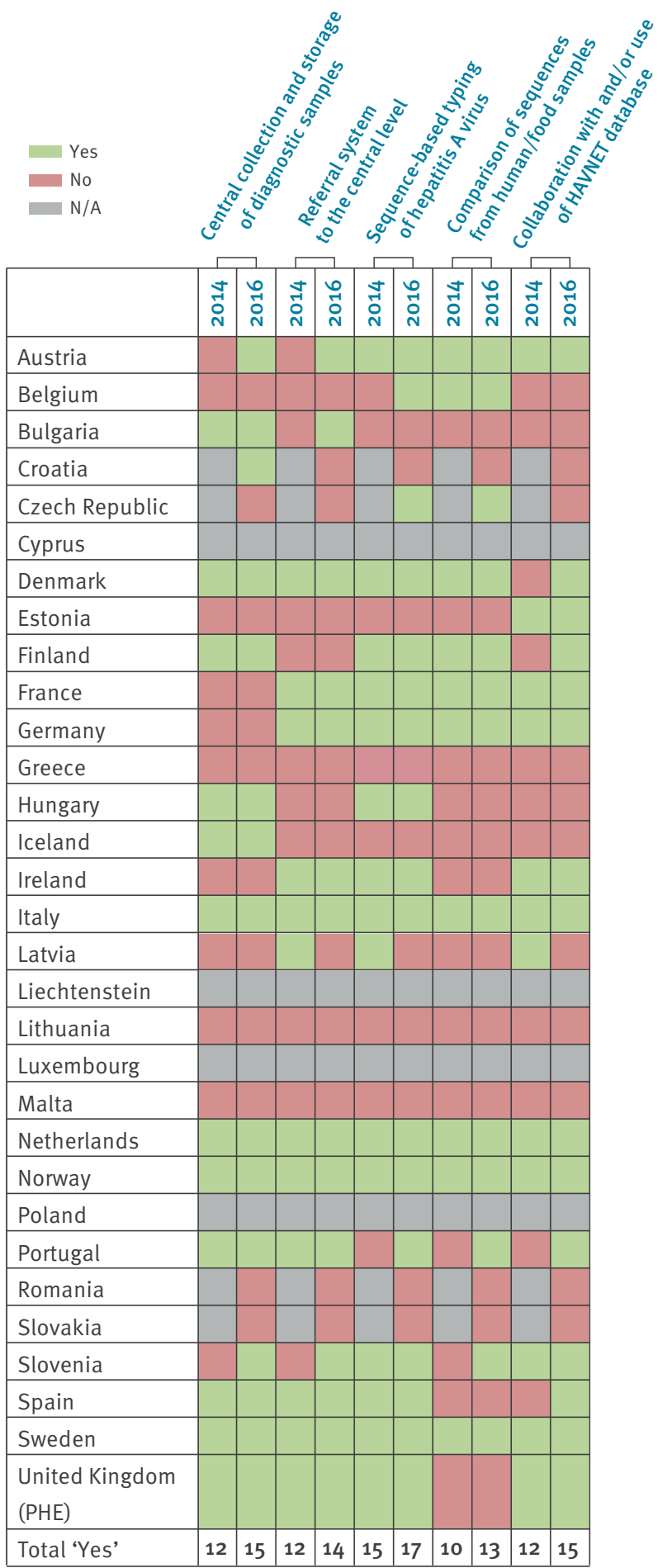

PHE: Public Health England; UK: United Kingdom.

the sequencing of a common consensus sequencing region, spanning the HAV VP1/2A junction, and thus promoting the sequence target region described in the HAVNET protocol [27]. In 2016, ECDC followed-up with a second survey to assess change in sequencing and some of the surveillance practices over time. Our study describes the analysis of the results from these surveys and assesses the progress made between 2014 and 2016 in harmonisation of HAV typing practices.

\section{Methods}

\section{Questionnaires}

In September 2014, ECDC performed a rapid consultation among the $31 \mathrm{EU} / \mathrm{EEA}$ countries to mainly collect information on HAV genotyping practices through the National Focal Points for food- and waterborne diseases and zoonoses (Supplement 1). The results of this first online survey were discussed in the multidisciplinary HAV expert consultation meeting at ECDC in October 2014, where it was agreed to recommend a common consensus sequencing region and/or a standard protocol for HAV sequencing as proposed by HAVNET [27]. HAVNET provides a laboratory protocol for the detection and sequencing of HAV, which includes instructions on RNA extraction, PCR settings, primers for the amplification of the $V P_{1} / 2 A$ junction, and reagents that can be used. In August 2016, ECDC distributed a followup questionnaire (Supplement 2) to assess if practices had changed or had become more compatible between countries.

The responses to both questionnaires were requested by October 2014 and October 2016, respectively.

Both surveys focused on laboratory methods used at the national level for sequence-based typing of HAV. In 2014, the sequence target region for typing and/or the protocol used for sequencing were queried. Although these were not initially investigated by the 2016 survey, several countries nonetheless provided information about their sequencing protocol or sequence target region. To complete knowledge on this, countries that had not covered this topic were contacted via email after the deadline of the 2016 survey.

The surveys also included questions about molecular surveillance practices related to sequence-based typing. These questions aimed at gaining knowledge on the central collection and storage of diagnostic samples of patients fulfilling the EU hepatitis A case definition (IgM or HAV RNA positive, with clinical criteria of an acute hepatitis) [28], and on the presence of a referral system for confirmation of primary diagnostics or for characterisation by sequencing (e.g. genotyping). Furthermore, countries were asked about their sequencing sampling strategy during outbreak situations.

Further questions in both surveys were on collaborations with the food sector on comparing sequences obtained from humans and/or food samples (food safety authorities and reference laboratories for food) and on collaborations with HAVNET in depositing and/ or comparing data in the HAVNET database. 
Central sample collection and place of storage, presence of a referral system for hepatitis A virus by country, European Union/European Economic Area, 2014 ( $n=23$ countries providing information) and 2016 ( $n=27$ countries providing information)

\begin{tabular}{|c|c|c|c|c|}
\hline \multirow{2}{*}{ Countries } & \multicolumn{2}{|c|}{ Central sample collection and place of storage } & \multicolumn{2}{|c|}{ Presence of a referral system } \\
\hline & $2014(n=12 / 23)$ & $2016(n=15 / 27)$ & $2014(n=12 / 23)$ & $2016(n=14 / 27)$ \\
\hline Austria & No & $\begin{array}{c}\text { National reference laboratory at the } \\
\text { Center for Virology, Medical University of } \\
\text { Vienna, Vienna }\end{array}$ & No & Yes, mandatory \\
\hline Belgium & No & No & No & No \\
\hline Bulgaria & $\begin{array}{l}\text { National reference laboratory at the } \\
\text { National Centre of Infectious and } \\
\text { Parasitic Diseases, Sofia }\end{array}$ & $\begin{array}{c}\text { National reference laboratory at the } \\
\text { National Centre of Infectious and Parasitic } \\
\text { Diseases, Sofia }\end{array}$ & No & Yes, voluntary \\
\hline Croatia & NA & $\begin{array}{c}\text { University Hospital for Infectious } \\
\text { Diseases, Zagreba }\end{array}$ & NA & No \\
\hline Czech Republic & NA & No & NA & No \\
\hline Denmark & $\begin{array}{l}\text { Virus Surveillance and Research } \\
\text { section at Statens Serum Institute, } \\
\text { Copenhagen }\end{array}$ & $\begin{array}{l}\text { Virus Surveillance and Research section } \\
\text { at Statens Serum Institute, Copenhagen }\end{array}$ & Yes, voluntary & Yes, voluntary \\
\hline Estonia & No & No & No & No \\
\hline Finland & $\begin{array}{l}\text { National Institute of Health and } \\
\text { Welfare, Helsinki }\end{array}$ & $\begin{array}{c}\text { National Institute of Health and Welfare, } \\
\text { Helsinki }\end{array}$ & No & No \\
\hline France & No & No & \multicolumn{2}{|c|}{$\begin{array}{l}\text { Yes, voluntary but mandatory during } \\
\text { outbreaks/detection of clusters (2014 } \\
\text { and 2016) }\end{array}$} \\
\hline Germany & No & No & Yes, voluntary & Yes, voluntary \\
\hline Greece & No & No & No & No \\
\hline Hungary & $\begin{array}{l}\text { National Public Health Institute, } \\
\text { Budapest }\end{array}$ & National Public Health Institute, Budapest & No & No \\
\hline Iceland & $\begin{array}{l}\text { Department of Virology, Landspitali } \\
\text { - National University Hospital, } \\
\text { Reykjavik }\end{array}$ & $\begin{array}{l}\text { Department of Virology, Landspitali - } \\
\text { National University Hospital, Reykjavik }\end{array}$ & No & No \\
\hline Ireland & No & No & Yes, voluntary & Yes, voluntary \\
\hline Italy & $\begin{array}{l}\text { National Reference Laboratory of the } \\
\text { Istituto Superiore di Sanità, Rome }\end{array}$ & $\begin{array}{l}\text { National Reference Laboratory of the } \\
\text { Istituto Superiore di Sanità, Rome }\end{array}$ & Yes, voluntary & Yes, voluntary \\
\hline Latvia & No & No & Yes, voluntary & No \\
\hline Lithuania & No & No & No & No \\
\hline Malta & No & No & No & No \\
\hline Netherlands & $\begin{array}{l}\text { National public health institute, } \\
\text { Bilthoven }\end{array}$ & National public health institute, Bilthoven & Yes, voluntary & Yes, voluntary \\
\hline Norway & $\begin{array}{c}\text { Norwegian Institute of Public Health, } \\
\text { Oslo }\end{array}$ & Norwegian Institute of Public Health, Oslo & Yes, mandatory & Yes, mandatory \\
\hline Portugal & $\begin{array}{l}\text { The National Institute of Health, } \\
\text { Lisbon }\end{array}$ & The National Institute of Health, Lisbon & Yes, voluntary & Yes, voluntary \\
\hline Romania & NA & No & NA & No \\
\hline Slovakia & NA & No & NA & No \\
\hline Slovenia & No & $\begin{array}{l}\text { Institute of Microbiology and } \\
\text { Immunology, Faculty of Medicine, } \\
\text { University of Ljubljana, Ljubljana }\end{array}$ & No & Yes, voluntary \\
\hline Spain & $\begin{array}{l}\text { National Center of Microbiology, } \\
\text { Institute of Health Carlos III, Madrid }\end{array}$ & $\begin{array}{c}\text { National Center of Microbiology, Institute } \\
\text { of Health Carlos III, Madrid }\end{array}$ & Yes, voluntary & Yes, voluntary \\
\hline Sweden & $\begin{array}{c}\text { Public Health Agency of Sweden } \\
\text { (PHAS), Solnaa and/or Sahlgrenska } \\
\text { hospital, Gothenburga }\end{array}$ & $\begin{array}{c}\text { Public Health Agency of Sweden (PHAS), } \\
\text { Solna and/or Sahlgrenska hospital, } \\
\text { Gothenburga }\end{array}$ & Yes, voluntary & Yes, voluntary \\
\hline $\begin{array}{l}\text { United Kingdom } \\
\text { (PHE) }\end{array}$ & $\begin{array}{l}\text { Virus Reference Department, PHE, } \\
\text { Colindale }\end{array}$ & $\begin{array}{l}\text { Virus Reference Department, PHE, } \\
\text { Colindale }\end{array}$ & Yes, voluntary & Yes, voluntary \\
\hline
\end{tabular}

NA: data not available; PHE: Public Health England.

${ }^{a}$ Only those samples that were also tested there by primary diagnostic or sequencing. 
European Union/European Economic Area countries performing sequence-based typing of hepatitis A virus in (A) 2014 $(n=23$ countries providing information) and (B) $2016(n=27$ countries providing information)

$$
\begin{aligned}
& \text { A. } 2014 \\
& \text { Yes }(n=15) \\
& \text { No }(n=8) \\
& \text { Data not available }(n=8)
\end{aligned}
$$

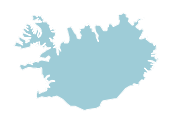

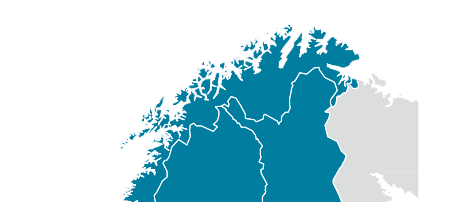

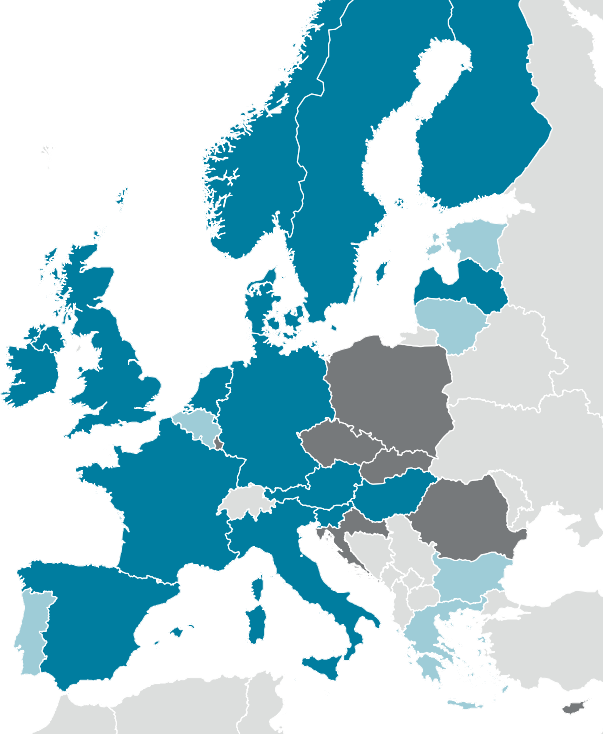

\section{B. 2016}

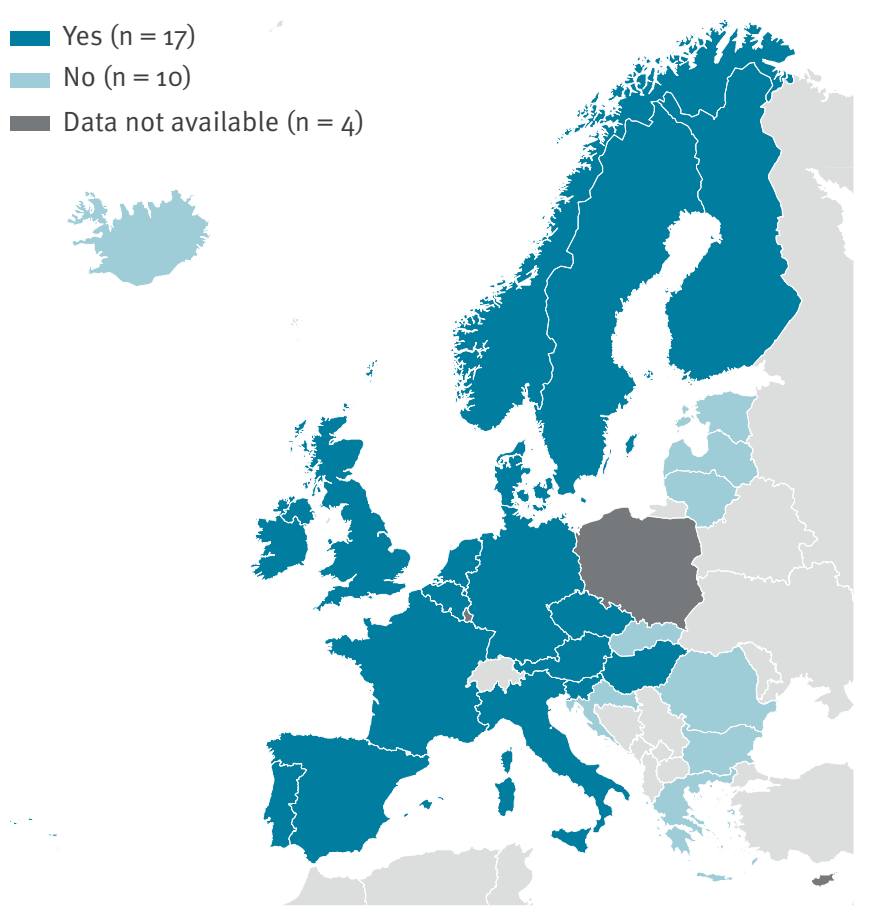

In the 2016 survey, additional information was collected on the timeliness of sequence submission to HAVNET in 2015.

\section{Ethical statement}

Ethical approval was not necessary for this study.

\section{Data analysis}

Counts and proportions of country responses to survey questions were calculated using Excel. Maps were generated using ECDC Map Maker (EMMa) [29]. Alignments of the amplified regions of the HAV genome were done using CLC Genomics and the complete HAV genome sequence (GenBank accession number: NC_001489) was used as reference. A schematic representation of the alignment was drawn using PowerPoint.

We present a descriptive analysis of the responses to the surveys and compare the sequence-based typing practices for HAV reported by EU/EEA countries in 2014 and 2016.

\section{Results}

\section{Survey participation}

In 2014,23 of 31 EU/EEA countries (74\%) responded to the survey. In 2016, four more countries (Croatia, Czech Republic, Romania, Slovakia) replied to the survey (27/31) resulting in a participation of $87 \%$. In both years, replies for the United Kingdom (UK) were provided by Public Health England and related to the practices ongoing in England, Northern Ireland and Wales. An overview of the countries participating in the surveys and procedural changes implemented over time is represented in Figure 1, with a total of 13 such changes visible.

Sample collection, storage and referral system In 2014,12 of 23 countries indicated central collection and storage of their samples defined as HAV positive by primary diagnostics and intended for sequencing, mostly at national level. Twelve countries had a referral system for the confirmation of the primary diagnostics or for characterisation by sequencing which was voluntary in 10 and mandatory in two countries (one in outbreak situation only).

The number of countries with central collection and storage of HAV positive samples was 15 in 2016, whereas the number of countries with a referral system for confirmation of samples increased by two $(n=14)$ compared to 2014 . Eleven countries reported a voluntary and three a mandatory system (one in outbreak situation only).

Austria and Slovenia reported no central collection and storage of HAV positive samples in 2014 but did so by the time of the second survey in 2016, as did Croatia, which had not participated in the 2014 survey. Austria, Bulgaria and Slovenia did not have a referral system in 2014 but all three countries established one by 2016. In contrast, Latvia had a referral system in 2014 with 
Hepatitis A virus genome and targets used for sequence-based typing, European Union/European Economic Area countries, 2014 ( $n=15$ countries providing information) and $2016(n=17$ countries providing information)
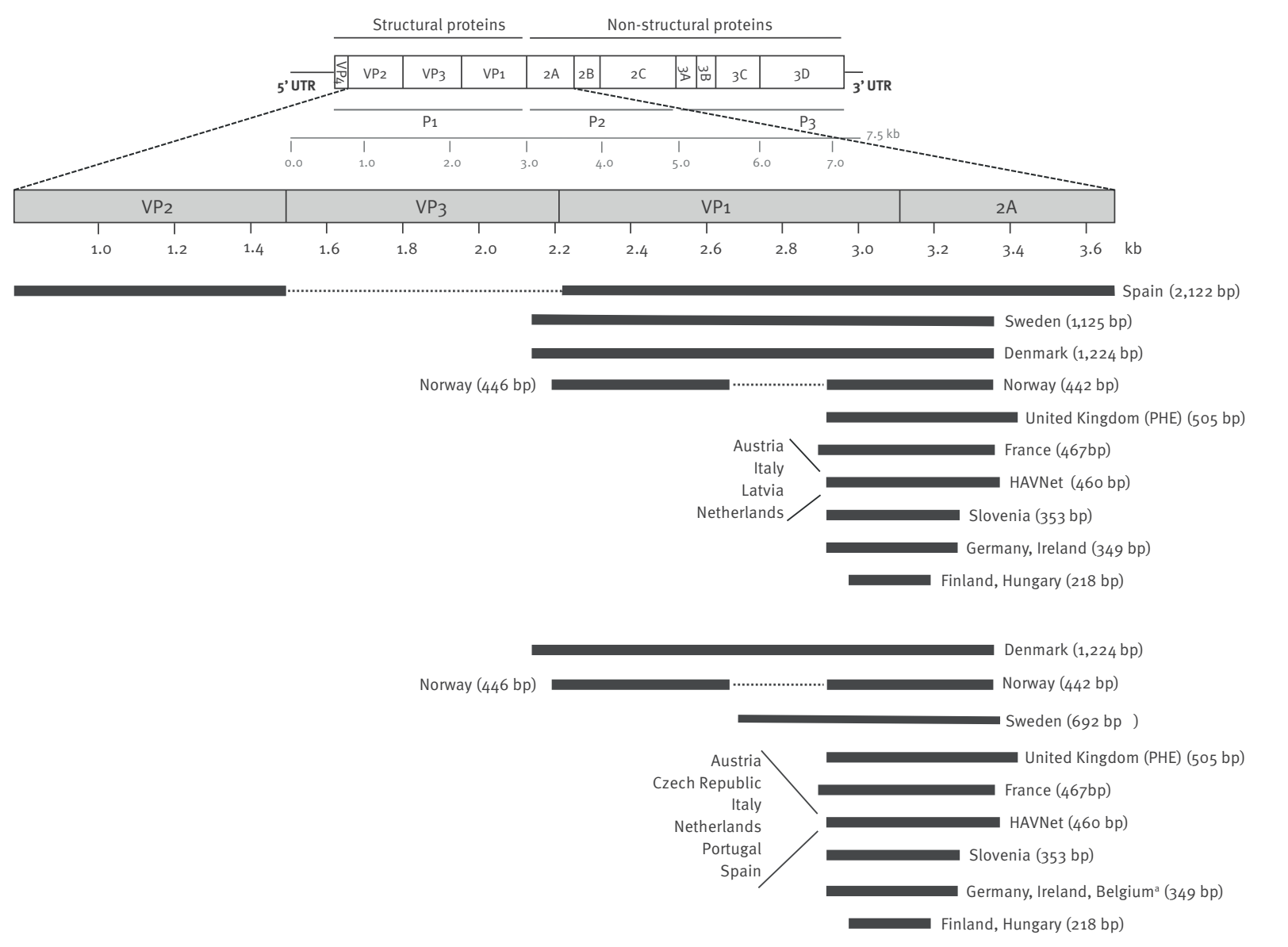

bp: base pair; kb: kilo bases; PHE: Public Health England; SSRNA: single stranded RNA; UTR: untranslated region; VP: viral protein.

${ }^{a}$ Belgium changed the region of the genome targeted for amplification/sequencing after 2016 and now uses the HAVNET protocol.

The $(+)$ SSRNA $(7.5 \mathrm{~kb})$ consists of three open reading frames $\left(P_{1}-P_{3}\right)$ encoding structural $\left(P_{1}\right)$ and non-structural proteins $\left(P_{2}\right.$, $\left.P_{3}\right)$. $P_{1}$ encodes the capsid polypeptides $\left(\mathrm{VP}_{1}-4\right)$, and the $\mathrm{P}_{2}$ and $\mathrm{P}_{3}$ regions encode the non-structural polypeptides required for virus replication. The horizontal bars represent the position in the genome of the region amplified and sequenced by the respective countries in 2014 and 2016. The sizes of the respective regions after removing primer binding sites are indicated in parentheses next to the horizontal bars. The GenBank sequence NC_001489 (HAV, complete genome) was used as reference.

voluntary reporting but it was not the case any longer in 2016. In most countries, the national reference laboratories have a supporting role offering laboratory diagnostics or genotyping on request (e.g. Germany, Ireland, the Netherlands and Spain). In France, referral to the reference laboratory is mandatory only when clusters of cases or outbreaks are identified (Table).

\section{Sequencing practices in European Union/ European Economic Area countries} More than half of the EU/EEA countries performed sequence-based typing (15/23 in 2014 and $17 / 27$ in 2016) at their reference laboratory or collaborating centre (Figure $2 \mathrm{~A}$ and $\mathrm{B}$ ). Latvia performed sequencing in 2014 but stopped it by 2016. While no information was obtained for the Czech Republic in 2014, it was confirmed during the second survey in 2016 that sequencing had been implemented in this country after 2014. Thus a total of three countries (Belgium, the Czech Republic and Portugal) started sequence-based typing after 2014. Of eastern EU/EEA countries, the Czech Republic and Hungary reported having capacity for sequencing and its implementation.

The sequenced genome regions and their length are shown in Figure 3. All of the responding EU/EEA countries $(n=15 / 15$ in 2014 and $n=17 / 17$ in 2016) sequence the $\mathrm{VP}_{1} / 2 \mathrm{~A}$ junction but the sequence length varies, with a minimum overlap of $218 \mathrm{nt}$ for both years. Three countries (Denmark, Norway; Sweden before 2016) sequence the $5^{\prime}$ end of $V_{1}$ in addition to the $\mathrm{VP}_{1} / 2 \mathrm{~A}$ junction. One country (Spain) used the VP1 and VP2 
region in addition to $\mathrm{VP}_{1} / 2 \mathrm{~A}$ for amplification before 2016. Four countries used the HAVNET protocol in 2014 and the numbers increased to six in 2016.

\section{Collaborations with the food sector and HAVNET}

Ten of 23 countries reported collaborations with the food sector for comparing sequences in 2014 and 13 of 27 in 2016. Portugal and Slovenia started to collaborate with the food sector after the 2014 survey. Of the countries joining the survey in 2016, the Czech Republic reported comparing sequences with the food sector (Figure $4 \mathrm{~A}$ and $\mathrm{B}$ ). Belgium stated that this is not done on a routine basis but only for outbreak investigations.

The number of countries reporting collaborations with HAVNET increased between the two surveys in 2014 $(n=12 / 23)$ and $2016(n=15 / 27)$. Latvia reported collaboration with HAVNET in 2014, but no longer in 2016. Denmark, Finland, Portugal and Spain started collaborating with HAVNET after 2014 (Figure $4 \mathrm{C}$ and D).

The majority of the countries reporting collaboration with HAVNET in 2016 submitted sequences to the database or used it for comparison of sequences. Of the countries submitting sequences, only three indicated a regular sequence submission (the Netherlands, Portugal and Sweden). The remaining countries only submitted in case of outbreak suspicion or on a nonregular basis (Austria, Finland, Germany, Ireland, Norway, and the UK). Five countries provided an answer about the time delay between sequencing and submission of results to HAVNET, Sweden stated that submission of sequences occurred immediately after results were obtained. For the other four replying countries (Germany, Norway, Portugal, and the UK), the time delays differed significantly, ranging from 30 days to several years, depending on the country capacities and prioritisations.

\section{Discussion}

The two surveys performed in 2014 and 2016 provide insights into the molecular surveillance and sequencing practices within the EU/EEA and assess the changes introduced following a large food-borne outbreak in 2013-14. Joint practices such as a common target region for sequencing suggested in the ECDC multidisciplinary HAV expert meeting in 2014, allow the comparison of results and therefore provide information for action. Our analysis identified some progress in this respect between 2014 and 2016, with most countries reporting positive developments in different aspects of sequencing practices. In total, 13 procedural changes were observed, indicating an improvement in preparedness and outbreak response capacity in Europe (Figure 1): the number of countries with a central collection and storage of human HAV samples increased, as did the countries with a mandatory referral system, those performing sequencing, and those collaborating with the food sector and HAVNET. According to the replies in 2014 and 2016, sequence-based typing of HAV samples has been implemented in the majority of EU/EAA countries, except for most eastern EU countries. It is important to note that six EU/EEA countries (Croatia, Estonia, Iceland, Latvia, Lithuania, Malta) report a mean of $<20$ confirmed hepatitis A cases per year in the period 2014-16, which makes the implementation of sequencing not a priority for these countries [6]. Only four countries did not provide information in both surveys.

One of the most important findings is that there is capacity for sequencing in the majority of the EU/ EEA countries allowing epidemiologically meaningful comparison of results with all countries performing sequencing of an overlapping region of the HAV genome (Figure 3).

Although reporting of hepatitis A was compulsory in all the 23 countries responding to the survey in 2014, systematic surveillance of HAV infections could be further improved in the majority of EU/EEA countries. The absence of a centralised sample collection or a referral system (in 12 and 13 countries in 2016, respectively) makes it challenging to compare HAV sequences, even on the national level.

The crucial role of sequence data analysis to investigate outbreaks and define transmission pathways has been demonstrated several times $[10,19,30]$. With a hepatitis A incubation period of about 1 month, it is challenging to link apparently sporadic cases and identify transmission mechanisms and/or vehicles of infection based only on patients' interviews. Sequencing analysis of HAV isolated from patients supports linking cases, revealing infection routes and identifying the vehicle of infection in food-borne outbreaks. In 2013, Italy was the most affected EU/EEA country in a large food-borne hepatitis A outbreak with >1,200 reported cases. Sequencing was described as having a key role in delineating this outbreak, linking cases lacking any epidemiological link and identifying the outbreak strain in the suspected food vehicle. Sequencing also revealed the circulation of other unrelated strains [20]. Greece performed sequencing during a hepatitis A outbreak among refugees, asylum seekers and migrants in 2016, demonstrating that cases were imported and not locally acquired [31]. In 2018, sequencing revealed a link between cases in Sweden and Austria during an HAV outbreak and confirmed imported frozen strawberries as the vehicle of infection in Sweden. Consequently, the strawberries were withdrawn from the market, which led to the end of the outbreak in Sweden [32]. The importance of a routine surveillance system, including prompt referral and typing of HAV samples, was also highlighted in a Dutch study published in 2014 [33]. The authors pointed out that large outbreaks are rapidly detected because of the large number of patients, but for the detection of slowly spreading and dispersed clusters molecular typing is essential. In addition to providing links between cases otherwise not recognised, sequencing helps to distinguish sporadic cases from outbreak cases, 


\section{FIGURE 4}

Collaborations of the European Union/European Economic Area countries with the (A, B) food sector and (C, D) HAVNET in 2014 ( $\mathrm{n}=23$ countries providing information) and 2016 ( $\mathrm{n}=27$ countries providing information)

\section{A. Food sector collaborations 2014}

Yes $(n=10)$

No $(n=13)$

Data not available $(\mathrm{n}=8)$


\section{HAVNET collaborations 2014}

Yes $(n=12)$

No $(n=11)$

Data not available $(\mathrm{n}=8)$
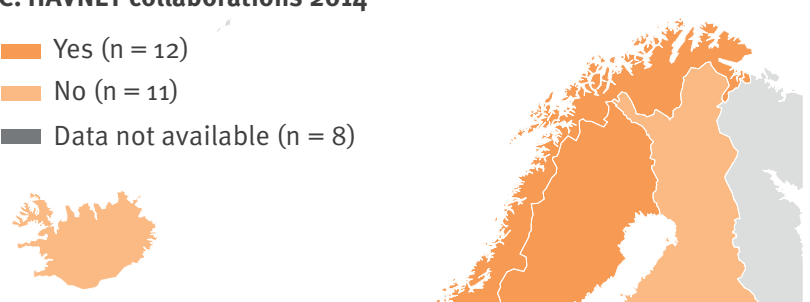

\section{HAVNET collaborations 2016}
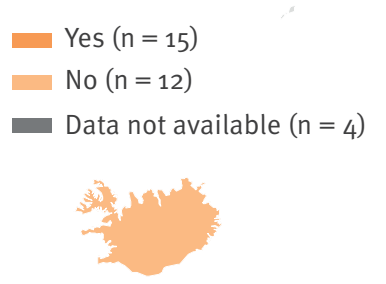

\section{B. Food sector collaborations 2016}


Countries collaborating with the food sector (food safety authorities and reference laboratories for food) on comparing sequences obtained from humans and/or food samples (A) in 2014 and (B) 2016 and countries collaborating with HAVNET (global network of hepatitis A

laboratories for sharing molecular and epidemiological data on hepatitis A - www.havnet.nl) in depositing and/or comparing data in the HAVNET database (C) in 2014 and (D) 2016. 
which improves the efficiency of epidemiological studies, and is essential to verify the end of an outbreak. Nevertheless, HAV sequence analysis is most useful to support outbreak investigations when applied together with relevant associated epidemiological information.

Regarding the genomic region to be sequenced, protocols for HAV sequence-based typing differ within the EU/EEA region, with all responding countries sequencing at least a part of the $\mathrm{VP}_{1} / 2 \mathrm{~A}$ junction (minimum fragment of $218 \mathrm{nt}$ ). The overlapping fragment in the same selected genome region allows a minimal comparison of different strains between countries. The VP $1 / 2 \mathrm{~A}$ junction is known to be one of the most variable regions of the HAV genome and therefore allows good resolution when comparing sequences. In the early 1990s, the different HAV genotypes were initially defined based on a 168 -nt fragment of the $\mathrm{VP}_{1} / 2 \mathrm{~A}$ junction [23] but were re-classified in the beginning of 2000 based on the longer VP1 sequence (900 nt) [34]. Some HAV strains differ from each other in $>20 \%$ of the nt in the $\mathrm{VP}_{1} / 2 \mathrm{~A}$ region and studies demonstrated a correlation of genotype and subgenotype with geographical clustering $[23,35,36]$. Due to the high variability of this region and despite the short size (ca500 nt), genotyping results can be obtained and genetic relatedness among HAV strains determined [36]. For outbreak investigations, analysis of the variable VP $1 / 2 \mathrm{~A}$ sequence together with the epidemiological association is sufficient to follow transmission events [37].

In order to achieve more meaningful molecular typing support for surveillance of hepatitis $A$ in Europe and to be able to compare HAV strains circulating in different countries, sequencing protocols targeting the same region are required. The short target of the $V_{P} 1 / 2 A$ junction allows easy and fast sequencing at lower cost compared with sequencing of larger regions. Furthermore, it is the most commonly investigated region in outbreaks, allowing the comparison of large numbers of previously sequenced strains in public databases [20].

Analysis of sequencing data also offers substantial benefits for identification of the outbreak vehicle or source of infection. Since HAV infections are often food-borne, sequencing of food items and collaborations with the food sector are of paramount importance. Most (13/17) countries performing sequencing in 2016 indicated collaboration with the food sector and comparison of sequences detected in human and food samples. Although sequencing from food samples is challenging due to the low viral load, inefficient extraction from different food matrices, the long incubation period of hepatitis A (up to 6 weeks) and the resulting recall bias of food consumption, it is invaluable in tracing the source of infections and providing evidence for public health actions [33,38]. A large part of the eastern European countries, which report the majority of the hepatitis A cases in the EU, neither perform sequencing yet, nor have collaborations established with the food sector, with consequent limited knowledge of the strains circulating in those countries.

HAVNET provides a global database that offers good support for outbreak investigations, e.g. comparison of strains at national and EU/EEA level. The network consists of virologists from universities and public health institutes using an online password-protected database platform. The use of the database is on a 'give-and-take-basis' and involves the signing of a confidentiality agreement. The platform provides online analysis and visualisation tools such as basic local alignment search tool (BLAST), phylogeny and a geographical analysis tool [25]. So far, HAVNET offers the only centralised collection of sequencing data and a standard protocol for HAV sequencing from human samples which additionally enables the comparison with HAV strains from food samples. Molecular data can be exchanged, strains circulating in different countries rapidly identified and therefore outbreak responses to multi-country threats improved. Although HAVNET provides a useful tool for outbreak investigations, only 15 countries reported collaborations with HAVNET in 2016.

To our knowledge, this is the first time information has been systematically gathered on the variety of existing laboratory practices supporting hepatitis A surveillance within the EU/EEA. Study limitations include the survey participation: despite a good participation, four countries did not reply to both surveys. Although some countries did not have sequencing capacity, they reported having access to it or collaborating with other countries during hepatitis A outbreaks. Greece, Latvia, Luxembourg and Malta performed sequencing in 2017 in the context of the hepatitis A outbreak mostly affecting MSM [15]. In Estonia, samples were referred to another laboratory outside the country (National Institute for Public Health and the Environment in the Netherlands) and Bulgaria sent its samples for sequencing to the Istituto Superiore di Sanità in Italy for ad hoc scientific collaborations [39]. A further limitation is that the question about the sequencing protocol was asked in 2014 only and the typing protocol may be different nowadays. However, countries had the opportunity to indicate changes in their sequencing practices and for the countries indicating to have started sequencing after 2014, additional information was collected separately. Spain decided to sequence 460 nt of the $\mathrm{VP}_{1} / 2 \mathrm{~A}$ junction (corresponding to the HAVNET protocol) instead of complete VP1, VP2 and $2 \mathrm{~A}$ after 2016 due to the high number of cases related to the European HAV outbreak disproportionally affecting MSM [15]. Sweden changed its protocol after the expert meeting in 2014 to cover the $460 \mathrm{nt}$ fragment suggested in the HAVNET protocol plus $232 \mathrm{nt}$ of the 3' $\mathrm{VP}_{1}$ region. Belgium replaced its sequencing protocol with the HAVNET protocol after 2016. Therefore, the results might slightly underestimate the true sequencing capacity to support hepatitis A surveillance in the EU/EEA. 
In conclusion, the surveys highlighted that many of the EU/EEA countries perform sequencing with rather variable procedures, but their sequenced regions are overlapping and thus comparable for surveillance purposes. Nonetheless, European collaboration in sharing and comparing HAV sequences on circulating strains in a timely manner can be further strengthened. Resulting collaborations could contribute significantly to accelerate public health responses and thereby stop transmission chains across borders and reduce the hepatitis A incidence. The population susceptibility to HAV infection in the EU/EEA has increased due to improved hygiene and sanitation that, together with the availability of a safe and effective vaccine, result in decreased virus circulation. However, hepatitis A surveillance in the EU/EEA is essential since the high population susceptibility in most of the countries, coupled with the high population mobility and single food market, increase the potential for cross-borders outbreaks. HAV sequencing is necessary to ensure the most efficient and timely response to such threats across the EU.

The application of whole genome sequencing (WGS) has increased within recent years and has become the reference molecular typing method in outbreak investigations [40]. Applying WGS to the detection of pathogens provides several advantages such as fast turn-around time and reduction of costs and workload. A high discrimination between HAV strains can be achieved since WGS allows the detection of minor variants. In the coming years, WGS might replace the sequencing of the $\mathrm{VP}_{1} / 2 \mathrm{~A}$ region. In the meantime, a higher degree of harmonisation in amplifying the $\mathrm{VP}_{1} / 2 \mathrm{~A}$ region will help to improve preparedness and accelerate public health response in Europe.

\section{Acknowledgements}

We have received valuable comments to the manuscript from Mike Catchpole, Piotr Kramaz and Marc Struelens and acknowledge their contribution in improving the paper.

\section{Conflict of interest}

None declared.

\section{Authors' contributions}

TE and KL analysed and validated the data, TE drafted the manuscript. ES, JT and KL designed the questionnaire, initiated, and developed the study. HV, LT, JD, MLB, ARC, RB, IC, SLN, KB, VN, MK, MT, AH, RK, AL, AA, MMC, RDS, DJ, JE, SK, VS, SWA, HH, KM, JLE, LS, AMRA, SKF, MP, LV, KSJ, SM, TKF, $M F$ and JJW contributed to the concept of the manuscript and reviewed it critically. All authors have read and approved the final manuscript.

\section{References}

1. World Health Organization (WHO). WHO position paper on hepatitis A vaccines - June 2012. Wkly Epidemiol Rec. 2012;87(28/29):261-76. PMID: 22905367

2. Vos T, Allen C, Arora M, Barber RM, Bhutta ZA, Brown A, et al. GBD 2015 Disease and Injury Incidence and Prevalence Collaborators. Global, regional, and national incidence, prevalence, and years lived with disability for 310 diseases and injuries, 1990-2015: a systematic analysis for the Global Burden of Disease Study 2015. Lancet. 2016;388(10053):1545 602. https://doi.org/10.1016/S0140-6736(16)31678-6 PMID: 27733282

3. Jacobsen KH, Wiersma ST. Hepatitis A virus seroprevalence by age and world region, 1990 and 2005 . Vaccine. 2010;28(41):6653-7. https://doi.org/10.1016/j. vaccine.2010.08.037 PMID: 20723630

4. Jeong SH, Lee HS. Hepatitis A: clinical manifestations and management. Intervirology. 2010;53(1):15-9. https://doi. org/10.1159/000252779 PMID: 20068336

5. European Centre for Disease Prevention and Control (ECDC). Hepatitis A. In: ECDC. Annual epidemiological report for 2016. Stockholm: ECDC; 2019. Available from: https://ecdc.europa. eu/sites/portal/files/documents/AER_for_2016-hepatitis-A_o. pdf

6. European Centre for Disease Prevention and Control (ECDC). Surveillance Atlas of Infectious Diseases. Stockholm: ECDC; 2019. Available from: https://ecdc.europa.eu/en/ surveillance-atlas-infectious-diseases

7. Carrillo-Santisteve P, Tavoschi L, Severi E, Bonfigli S, Edelstein M, Byström E, et al. ECDC HAV Expert Panel. Seroprevalence and susceptibility to hepatitis A in the European Union and European Economic Area: a systematic review. Lancet Infect Dis. 2017;17(10):e306-19. https://doi.org/10.1016/S14733099(17)30392-4 PMID: 28645862

8. Beauté J, Westrell T, Schmid D, Müller L, Epstein J, Kontio M, et al. Travel-associated hepatitis A in Europe, 2009 to 2015. Euro Surveill. 2018;23(22):1700583. https://doi.org/10.2807/15607917.ES.2018.23.22.1700583 PMID: 29871720

9. Sane J, MacDonald E, Vold L, Gossner C, Severi E, on behalf of the International Outbreak Investigation Team. Multistate foodborne hepatitis A outbreak among European tourists returning from Egypt--need for reinforced vaccination recommendations, November 2012 to April 2013. Euro Surveill. 2015;20(4):21018. https://doi.org/10.2807/1560-7917. ES2015.20.4.21018 PMID: 25655054

10. MacDonald E, Steens A, Stene-Johansen K, Gillesberg Lassen S, Midgley S, Lawrence J, et al. Increase in hepatitis A in tourists from Denmark, England, Germany, the Netherlands, Norway and Sweden returning from Egypt, November 2012 to March 2013. Euro Surveill. 2013;18(17):20468. PMID: 23647624

11. Werber D, Michaelis K, Hausner M, Sissolak D, Wenzel J, Bitzegeio J, et al. Ongoing outbreaks of hepatitis A among men who have sex with men (MSM), Berlin, November 2016 to January 2017 - linked to other German cities and European countries. Euro Surveill. 2017;22(5):30457. https://doi. org/10.2807/1560-7917.ES.2017.22.5.30457 PMID: 28183391

12. Beebeejaun K, Degala S, Balogun K, Simms I, Woodhall SC, Heinsbroek E, et al. Outbreak of hepatitis A associated with men who have sex with men (MSM), England, July 2016 to January 2017. Euro Surveill. 2017;22(5):30454. https://doi. org/10.2807/1560-7917.ES.2017.22.5.30454 PMID: 28183392

13. Freidl GS, Sonder GJ, Bovée LP, Friesema IH, van Rijckevorsel GG, Ruijs WL, et al. Hepatitis A outbreak among men who have sex with men (MSM) predominantly linked with the EuroPride, the Netherlands, July 2016 to February 2017. Euro Surveill. 2017:22(8):30468. https://doi.org/10.2807/1560-7917. ES.2017.22.8.30468 PMID: 28251892

14. European Centre for Disease Prevention and Control (ECDC). Hepatitis A outbreaks in the EU/EEA mostly affecting men who have sex with men - third update, 28 June 2017. Stockholm: ECDC. 2017. Available from: https://ecdc.europa.eu/en/ publications-data/rapid-risk-assessment-hepatitis-outbreakeueea-mostly-affecting-men-who-have-sex

15. Ndumbi P, Freidl GS, Williams CJ, Mårdh O, Varela C, Avellón A, et al. Hepatitis A outbreak disproportionately affecting men who have sex with men (MSM) in the European Union and European Economic Area, June 2016 to May 2017. Euro Surveill. 2018;23(33):1700641. https://doi.org/10.2807/1560-7917. ES.2018.23.33.1700641 PMID: 30131095

16. Latash J, Dorsinville M, Del Rosso P, Antwi M, Reddy V, Waechter $\mathrm{H}$, et al. Notes from the Field: Increase in Reported Hepatitis A Infections Among Men Who Have Sex with Men New York City, January-August 2017. MMWR Morb Mortal Wkly Rep. 2017;66(37):999-1000. https://doi.org/10.15585/mmwr. mm6637a7 PMID: 28934181 
17. Rivas V, Barrera A, Pino K, Núñez R, Caceres CJ, Lopez-Lastra $M$, et al. Hepatitis A outbreak since November 2016 affecting men who have sex with men (MSM) in Chile connected to the current outbreak in MSM in Europe, situation up to October 2017. Euro Surveill. 2018;23(9):1800060. https:// doi.org/10.2807/1560-7917.ES.2018.23.9.18-00060 PMID: 29510780

18. Gozlan Y, Bar-Or I, Rakovsky A, Savion M, Amitai Z, Sheffer R, et al. Ongoing hepatitis A among men who have sex with men (MSM) linked to outbreaks in Europe in Tel Aviv area, Israel, December 2016 - June 2017. Euro Surveill. 2017;22(29):30575. https://doi.org/10.2807/1560-7917.ES.2017.22.29.30575 PMID: 28749336

19. Severi E, Verhoef L, Thornton L, Guzman-Herrador BR, Faber M, Sundqvist $L$, et al. Large and prolonged food-borne multistate hepatitis A outbreak in Europe associated with consumption of frozen berries, 2013 to 2014. Euro Surveill. 2015;20(29):21192. https://doi.org/10.2807/1560-7917.ES2015.20.29.21192 PMID: 26227370

20. Bruni R, Taffon S, Equestre M, Chionne P, Madonna E, Rizzo $C$, et al. Key Role of Sequencing to Trace Hepatitis A Viruses Circulating in Italy During a Large Multi-Country European Foodborne Outbreak in 2013. PLoS One. 2016;11(2):e0149642. https://doi.org/10.1371/journal.pone.0149642 PMID: 26901877

21. Costa-Mattioli M, Di Napoli A, Ferré V, Billaudel S, PerezBercoff R, Cristina J. Genetic variability of hepatitis A virus. J Gen Virol. 2003;84(Pt 12):3191-201. https://doi.org/10.1099/ vir.0.19532-0 PMID: 14645901

22. Lemon SM, Jansen RW, Brown EA. Genetic, antigenic and biological differences between strains of hepatitis A virus. Vaccine. 1992;10(Suppl 1):S40-4. https://doi. org/10.1016/0264-410X(92)90540-Z PMID: 1335657

23. Robertson BH, Jansen RW, Khanna B, Totsuka A, Nainan OV, Siegl G, et al. Genetic relatedness of hepatitis A virus strains recovered from different geographical regions. J Gen Virol. 1992;73(Pt 6):1365-77. https://doi.org/10.1099/0022-1317-736-1365 PMID: 1318940

24. Desbois D, Couturier E, Mackiewicz V, Graube A, Letort MJ, Dussaix E, et al. Epidemiology and genetic characterization of hepatitis A virus genotype IIA. J Clin Microbiol. 2010;48(9):3306-15. https://doi.org/10.1128/JCM.00667-10 PMID: 20592136

25. Kroneman A, de Sousa R, Verhoef L, Koopmans MPG, Vennema HOn Behalf Of The HAVNet Network. Usability of the international HAVNet hepatitis A virus database for geographical annotation, backtracing and outbreak detection. Euro Surveill. 2018;23(37):1700802. https:// doi.org/10.2807/1560-7917.ES.2018.23.37.1700802 PMID: 30229723

26. Aragonès L, Bosch A, Pintó RM. Hepatitis A virus mutant spectra under the selective pressure of monoclonal antibodies: codon usage constraints limit capsid variability. J Virol. 2008;82(4):1688-700. https://doi.org/10.1128/JVI.01842-07 PMID: 18057242

27. National Institute for Public Health and the Environment (RIVM). HAVNet. Bilthoven: RIVM. Available from: https://www. rivm.nl/documenten/typing-protocol-havnet-vp1p2a.

28. European Commission. Commission Regulation (EC) No $2018 / 945$ of 22 June 2018 on the communicable diseases and related special health issues to be covered by epidemiological surveillance as well as relevant case definitions under Decision No 1082/2013/EU of the European Parliament and of the Council of 22 October 2013. Official Journal of the European Union. Luxembourg: Publications Office of the European Union. 06/072018: L 170. Available from: https://eur-lex.europa.eu/ legal-content/EN/TXT/PDF/?uri=CELEX:32018Do945\&from=EN \#page $=22$

29. European Centre for Disease Control and Prevention (ECDC). ECDC Map Maker (EMMa). Stockhom: ECDC. [Accessed 18 Jan 2018]. Available from: https://emma.ecdc.europa.eu/

30. Nordic Outbreak Investigation Team C. Joint analysis by the Nordic countries of a hepatitis A outbreak, October 2012 to June 2013: frozen strawberries suspected. Euro Surveill. 2013;18(27):20520. https://doi.org/10.2807/1560-7917. ES2013.18.27.20520 PMID: 23870076

31. Mellou K, Chrisostomou A, Sideroglou T, Georgakopoulou T, Kyritsi M, Hadjichristodoulou C, et al. Hepatitis A among refugees, asylum seekers and migrants living in hosting facilities, Greece, April to December 2016. Euro Surveill. 2017;22(4):30448. https://doi.org/10.2807/1560-7917. ES.2017.22.4.30448 PMID: 28181904

32. Enkirch T, Eriksson R, Persson S, Schmid D, Aberle SW, Löf $E$, et al. Hepatitis A outbreak linked to imported frozen strawberries by sequencing, Sweden and Austria, June to September 2018. Euro Surveill. 2018;23(41):1800528. https://
doi.org/10.2807/1560-7917.ES.2018.23.41.1800528 PMID: 30326994

33. Petrignani M, Verhoef L, Vennema H, van Hunen R, Baas $D$, van Steenbergen JE, et al. Underdiagnosis of foodborne hepatitis A, The Netherlands, 2008-2010(1.). Emerg Infect Dis. 2014;20(4):596-602. https://doi.org/10.3201/eid2004.130753 PMID: 24655539

34. Costa-Mattioli M, Cristina J, Romero H, Perez-Bercof R, Casane $D$, Colina R, et al. Molecular evolution of hepatitis A virus: a new classification based on the complete VP1 protein. J Virol. 2002;76(18):9516-25. https://doi.org/10.1128/JVI.76.18.95169525.2002 PMID: 12186933

35. Brown EA, Jansen RW, Lemon SM. Characterization of a simian hepatitis A virus (HAV): antigenic and genetic comparison with human HAV. J Virol. 1989;63(11):4932-7. PMID: 2552172

36. Wang H, Zheng H, Cao J, Zhou W, Yi Y, Jia Z, et al. Genetic diversity of hepatitis A virus in China: VP3-VP1-2A genes and evidence of quasispecies distribution in the isolates. PLoS One. 2013;8(9):e74752. https://doi.org/10.1371/journal. pone.0074752 PMID: 24069343

37. Vaughan G, Xia G, Forbi JC, Purdy MA, Rossi LM, Spradling $P R$, et al. Genetic relatedness among hepatitis A virus strains associated with food-borne outbreaks. PLoS One. 2013;8(11):e74546. https://doi.org/10.1371/journal. pone.0074546 PMID: 24223112

38. Food and Drug Administration bacteriological analytical manual (BAM). Detection of hepatitis A in foods. BAM; [Accessed o8 Jan 2018]. Available from: http://www.fda.gov/ Food/FoodScienceResearch/LaboratoryMethods/ucm374006. htm

39. Bruni R, Taffon S, Equestre M, Cella E, Lo Presti A, Costantino $A$, et al. Hepatitis a virus genotypes and strains from an endemic area of Europe, Bulgaria 2012-2014. BMC Infect Dis. 2017;17(1):497. https://doi.org/10.1186/s12879-017-2596-1 PMID: 28705178

40. European Centre for Disease Prevention and Control (ECDC). Expert opinion on whole genome sequencing for public health surveillance. Stockholm: ECDC; 2016. Available from: https:// ecdc.europa.eu/sites/portal/files/media/en/publications/ Publications/whole-genome-sequencing-for-public-healthsurveillance.pdf

\section{License, supplementary material and copyright}

This is an open-access article distributed under the terms of the Creative Commons Attribution (CC BY 4.0) Licence. You may share and adapt the material, but must give appropriate credit to the source, provide a link to the licence and indicate if changes were made.

Any supplementary material referenced in the article can be found in the online version.

This article is copyright of the authors or their affiliated institutions, 2019. 